\title{
DIGITAL TECHNOLOGY The power of digital communications: improving outpatient attendances in south London
}

\author{
Authors: Mark Bartlett, ${ }^{A}$ Simon Blazer, ${ }^{B}$ Gina Hobson ${ }^{C}$ and Ian Abbs ${ }^{D}$
}

\begin{abstract}
Missed appointments represent a significant burden to healthcare budgets. The average 'did not attend' (DNA) rate across the NHS is $6.7 \%$; however, significant variation can be observed. In 2015, Guy's and St Thomas' NHS Foundation Trust observed a DNA rate of $13.5 \%$ and partnered with DrDoctor to reduce this by implementing their innovative outpatient scheduling and booking platform. The overall rate has reduced by $17.2 \%$; Guy's and St Thomas' NHS Foundation Trust has estimated a $£ 2.6$ million financial benefit in the first year and $91 \%$ of patients would recommend the service. This case study describes the onboarding process for DrDoctor's platform across Guy's and St Thomas' NHS Foundation Trust, the benefits delivered and next steps for the partnership.
\end{abstract}

KEYWORDS: Digital communications, outpatients scheduling, appointment management, technology, 'did not attend'

\section{Introduction}

NHS Digital reported between 2015 and 2016 over 7.5 million (6.7\% of the total) NHS outpatient appointments were missed in England. ${ }^{1}$ The rate at which appointments are missed is known as the 'did not attend' (DNA) rate and varies greatly across hospitals and clinics. Studies have shown that the effect of missing appointments creates inefficiencies by reducing the utilisation of clinical resources, increasing referral to treatment times, and inducing stress on staff from the added pressure of trying to reduce avoidable costs. ${ }^{2}$ While it is difficult to establish the exact financial impact of missed appointments, the health minister Jeremy Hunt has estimated that missed hospital appointments cost the NHS up to $£ 750$ million a year. ${ }^{3}$ Overbooking clinics can be used to compensate for missed appointments; however, this can cause chaos on the occasions when all patients attend. ${ }^{4}$

In order to reduce the effects of DNA rates, the company DrDoctor partnered with Frimley Health in 2012 to develop an innovative web-based platform to facilitate communication between hospitals and patients. Between 2012 and 2015 this

Authors: ${ }^{\text {A }}$ account manager, DrDoctor, London, UK; ${ }^{\text {Bhead of }}$ finance, Evelina London SBU, London, UK; ${ }^{C}$ communications associate, DrDoctor, London, UK; ${ }^{D}$ chief medical officer, Guy's and Saint Thomas' NHS Foundation Trust, London, UK platform was shared across three further trusts: Yeovil District Hospital, Nottingham University Hospital and Aneurin Bevan University Health Board.

Two months after implementation, DNA rates were reduced by $20-30 \%$ at each site. This has resulted in minimum cost savings of $£ 1$ million per site in increased efficiencies. ${ }^{5}$

Guy's and St Thomas' NHS Foundation Trust (GSTT) have 2.3 million patient contacts each year, 1.23 million of which are planned outpatient visits. ${ }^{6}$ In 2015 the DNA rate for new appointments at GSTT was $13.5 \%$, double the national average of $6.7 \%$, despite using a text message reminder service. Solutions were sought to reduce this and DrDoctor was piloted between May and December 2015 in the Women's Services directorate at GSTT, which has 2,500 outpatient appointments a month and a high DNA rate of $12.8 \%$. The initial results demonstrated a $34 \%$ reduction in DNAs equalling $£ 317,000$ increased revenue from activity; this led to the system being procured and implemented for all GSTT outpatient departments. The procurement process was completed in March 2016 and the project began in April 2016. The methodology and results of the GSTT project are described through the course of this paper.

\section{Methods}

The platform developed by DrDoctor enables patients to view their appointments online and access specific clinical and location information about their visit; this is supported by conversational SMS booking confirmations and reminder messages as shown in Fig 1. Patients can use the platform or conversational SMS to reach out to their hospital and reschedule or cancel appointments if they are unable to attend. Features, content and operational parameters, eg how many times a patient is given the opportunity to reschedule, are customised to the requirements of the hospital's internal policies and can be updated at any point.

To provide the above the following steps need to be delivered as an onboarding project:

> Integration - DrDoctor technically integrates with the hospital's Patient Administration System (PAS)

> Training - onboard staff members with different roles and responsibilities

> Go-Live - turn on functionality and provide support.

DrDoctor and GSTT planned the onboarding project to last 6 months and used PRINCE2 Agile project management structure to govern. ${ }^{8}$ This included regular team and board meetings run by the chief medical officer and the deputy director of operations to monitor progress and steer decision making. 

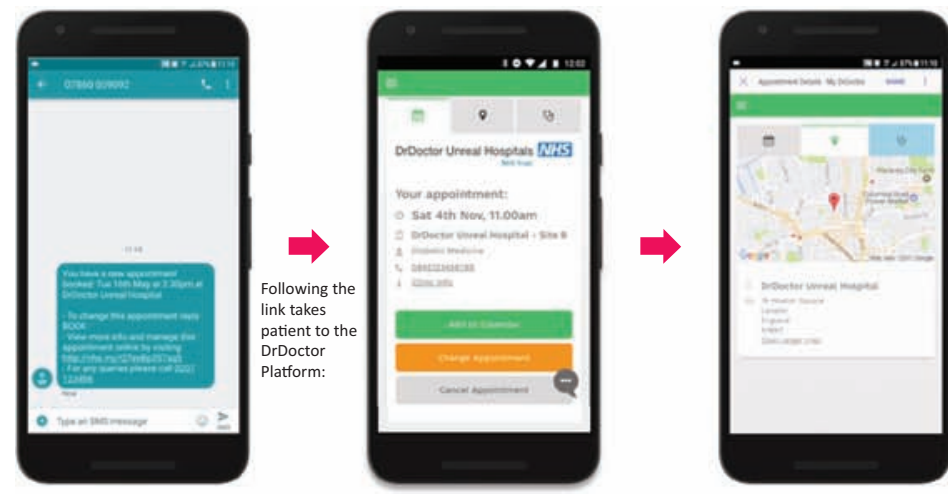
SMS confirmation and
reminders

DrDoctor uses i.PM data to send patients conversational SMS confirmations and reminders.

Each message has a unique URL to onboard patient to the platform.

\section{Landing page}

Overview of the appointment.

Patients can request to change/cancel an appointment and download a calendar reminder.
Location info page

Rich location information for the appointment is available.

Google Maps can be launched and hospitals can upload local maps.

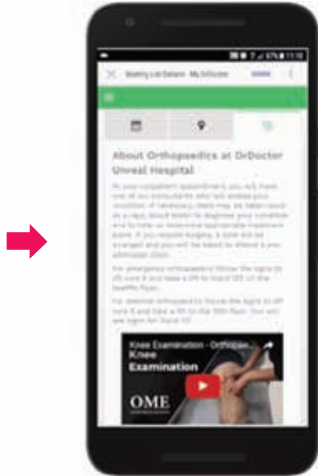

Clinical info page

Details of what to expect on the day helps patients prepare for their appointment.

Videos and images can be uploaded.

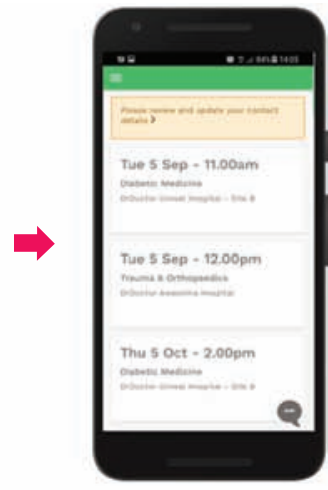

Holistic view

All upcoming appointments are available on the platform.

\section{Landing page,} location info page, and clinical info page area available for each appointment.

Patients can reply to reach out to the hospital if internet is not available.

Fig 1. Examples of the text messages patients receive, and the online patient portal where patients can view and reschedule their appointments online, and access appointment, location, and clinical information.

Information governance documentation was completed, assessed and signed off before the project commenced.

\section{Integration}

To integrate with the GSTT PAS (i.PM, supplied by DXC LTD), the GSTT information technology team built data views using standardised DrDoctor schemas provided. These data views are used by DrDoctor to pull the appointment information, which is grouped by clinic code then session codes. The GSTT and DrDoctor servers within the N3 (the national broadband network for the $\mathrm{NHS}$ ) network were linked and data were sent daily between the systems.

\begin{tabular}{lll}
$\begin{array}{l}\text { Table 1. Volume of staff trained at GSTT during the } \\
\text { project }\end{array}$ & Responsibility & $\begin{array}{l}\text { Number of staff } \\
\text { trained at GSTT }\end{array}$ \\
\hline Role & $\begin{array}{l}\text { Provide oversight and action } \\
\text { reporting }\end{array}$ & 20 \\
Lead & $\begin{array}{l}\text { Maintain content and system } \\
\text { Superuser }\end{array}$ & 142 \\
Botting & 224 \\
\hline
\end{tabular}

GSTT = Guy's and St Thomas' NHS Foundation Trust

\section{Training}

GSTT employs 15,000 staff and their outpatient services include 90 different specialties which are grouped into 14 different directorates. The priority list was discussed internally from analysis ordering by value of financial opportunity available (unpublished data). During onboarding GSTT staff were assigned roles as outlined in Table 1.

\section{Go-Live}

The DrDoctor functionality is grouped depending on session code, which gives superusers the ability to customise patient information to this detail. During the go-live process, superusers were given the responsibility to customise and turn on features at their discretion. A reporting process was developed to notify the team of completion rates and turn off the legacy reminder system in place.

\section{Table 2. DNA rates pre and post DrDoctor onboarding project for the whole of GSTT}

\begin{tabular}{lllll}
$\begin{array}{l}\text { Activity } \\
\text { type }\end{array}$ & $\begin{array}{l}\text { Baseline } \\
\text { DNA rate }\end{array}$ & $\begin{array}{l}\text { Post DrDoctor } \\
\text { DNA rate }\end{array}$ & $\begin{array}{l}\text { Change in } \\
\text { DNA rate }\end{array}$ & $\begin{array}{l}\text { Percentage } \\
\text { change }\end{array}$ \\
New & $13.0 \%$ & $11.0 \%$ & $2.0 \%$ & $15.5 \%$ \\
Follow-up & $13.8 \%$ & $11.3 \%$ & $2.5 \%$ & $17.7 \%$ \\
Total & $13.6 \%$ & $11.2 \%$ & $2.4 \%$ & $17.2 \%$ \\
\hline
\end{tabular}

DNA = 'did not attend', GSTT = Guy's and St Thomas' NHS Foundation Trust 


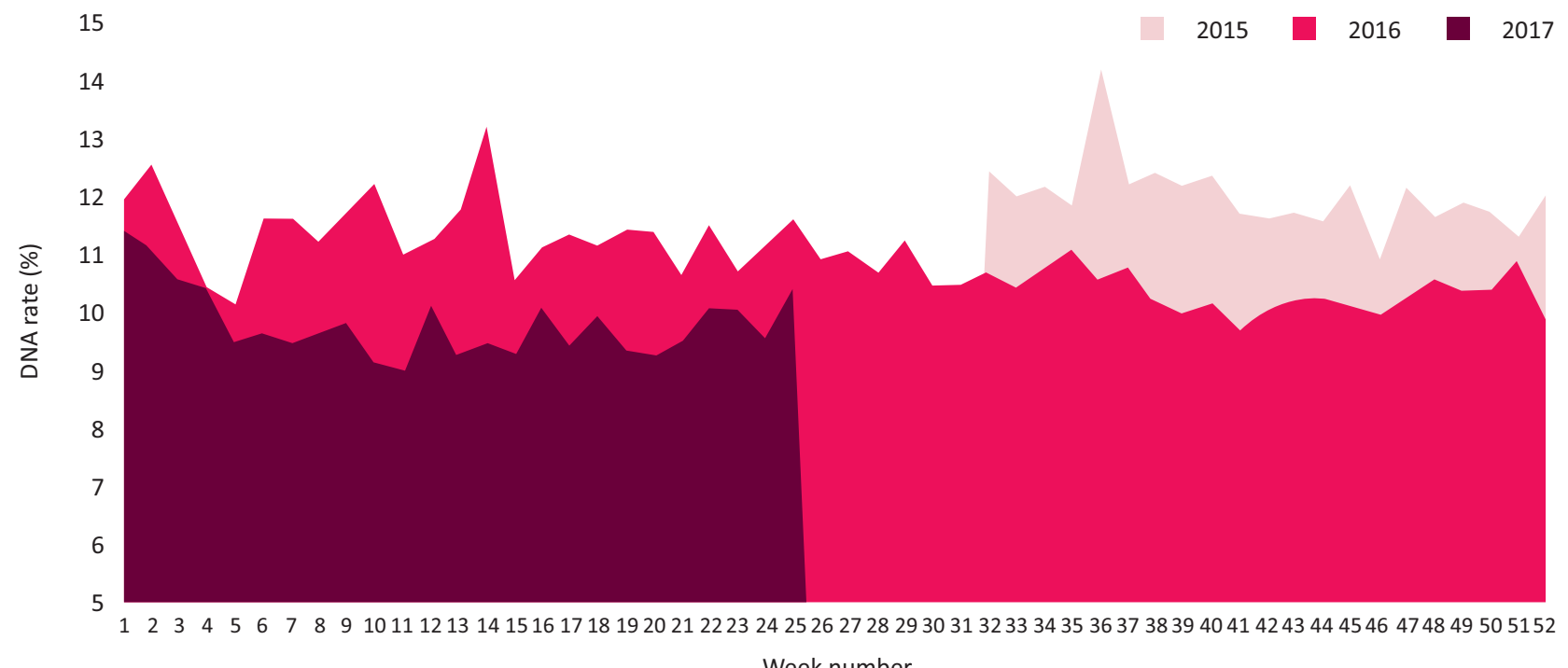

Fig 2. Weekly 'did not attend' (DNA) rates from August 2015 to July 2017 for the whole trust. The DrDoctor onboarding project started in week 14 2016 and completed week 402016 (post 30 September 2016). Post week 40 DNA rates have decreased by $17.2 \%$ for both new and follow-up appointments across the trust.

\section{Benefits}

The methodology used to assess the financial impact of the project was based on a comparison of DNA rates before, as calculated in the full business case (FBC), and after the implementation of DrDoctor at a specialty level. The DNA rate comparison was used to estimate the number of additional attendances (which would previously have been DNAs) and costs associated, using average specialty tariffs.

\section{Outcome}

Integration with i.PM was successfully completed in the pre-project phase. GSTT have a total of 10,578 session codes for customisation to go-live. By the end of the project on 30 September 2016 all required staff at GSTT were trained and onboarded to use DrDoctor, and $58 \%$ of session codes had reminder functionality switched on. Challenges were encountered during training and go-live phase, reducing the amount of codes activated. These were attributable

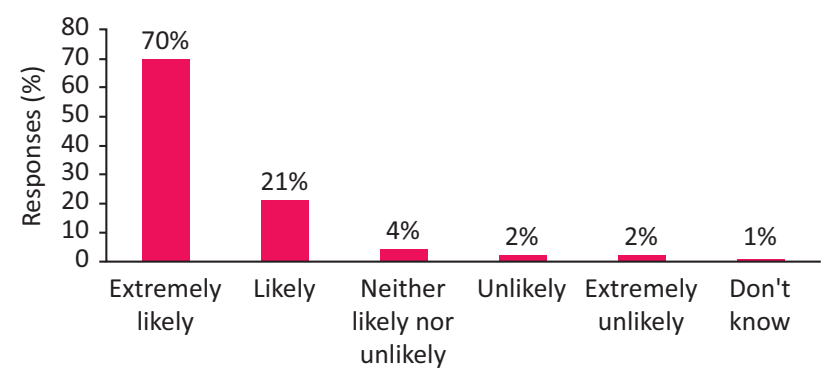

Fig 3. Patient responses to a friends and family test about the DrDoctor appointment platform. On 30 September 2016, patients who had received a text message and used the DrDoctor service were asked how likely they were to recommend to a family or friend and for further feedback. Of the 2945 patients who were asked, 788 responded. The results above show that $91 \%$ of patients were 'extremely likely' and 'likely' to recommend the service. to the diversity of pre-existing operational processes and use of IT systems across the different services at GSTT. ${ }^{9}$

At the end of the 6-month onboarding phase the DNA rates across the trust dropped from $11.7 \%$ to $10.4 \%$ (11.1\% reduction). Table 2 shows the rates as calculated for the 2016/2017 financial year with numbers in the $\mathrm{FBC}$ as the baseline. A year-on-year assessment of GSTT DNA rates from 2015-17, as calculated from data sent to DrDoctor, demonstrates a decrease from an average of $12.0 \%$ in 2015 to $9.8 \%$ in 2017 (Fig 2).

GSTT internal reporting has calculated the 2016/17 full year equivalent financial benefit for the trust as $£ 2.6$ million.

Data has also shown that one in five patients at GSTT now engage with their services digitally by following the link to their portal received in text message reminders. In response to a friends and family test about the service, of 788 responses $91 \%$ would recommend or highly recommend the service, see Fig 3.

\section{Conclusions}

The onboarding project was delivered on time, the $£ 2.6$ million financial benefit was in line with what was set out in the business case and DNA reductions were consistent with previous studies. ${ }^{10}$

The core platform for outpatients is being managed internally by the trust with ongoing support from the company. GSTT and DrDoctor continue to work in close partnership, which allows quick feedback and development iterations that are used to continually improve and tailor the product. Superusers tailor communications to the needs of different patient populations which can add further benefits. ${ }^{11,12}$

The trust has projected further savings of $£ 600,000-£ 650,000$ by increasing feature use to $80 \%$. The capabilities have extended to cover inpatient admissions and aim to include diagnostics, to give patients a holistic view of all appointments on their DrDoctor portal. A project to roll out further automated features is being piloted in the Dental directorate and has shown promising results. 
Since ending this project DrDoctor is being supported by DigitalHealth.London and the NHS Innovation Accelerator, and is working with two further London trusts.

Future healthcare needs to deliver despite increased demands and fewer resources, necessitating reviews of how resources are being used by initiatives in the NHS. ${ }^{13}$ Utilising clinics and staff time is an important part of the work. This project has demonstrated an increase in attendance, reduced waste whilst receiving positive patient feedback by moving beyond simple text message reminders and providing a responsive digital service.

The DrDoctor and GSTT partnership has the vision 'to be global leaders in digital patient communications and lead the NHS in digital patient services'. By delivering on this the aim is to improve the use of outpatient services and deliver exceptional care to patients.

\section{Conflicts of interest}

$\mathrm{MB}$ and $\mathrm{GH}$ are employed by DrDoctor, ICNH Ltd.

\section{Acknowledgements}

The authors would like to acknowledge the work of all the staff and service users at Guy's and St Thomas' NHS Foundation Trust and DrDoctor.

\section{References}

1 NHS Digital. Hospital Outpatient Activity 2015-16. www.digital. nhs.uk/catalogue/PUB22596

2 McLean SM, Booth A, Gee M et al. Appointment reminder systems are effective but not optimal: results of a systematic review and evidence synthesis employing realist principles. Patient Prefer Adherence 2016;10,479-99.

3 Torjesen I. Patients will be told cost of missed appointments and may be charged. BMJ 2015;351:h3663.

4 Dunnill MG, Pounder RE. Medical outpatients: changes that can benefit patients. Clin Med 2004;4:45-9.

5 Case Studies. www.drdoctor.co.uk/case-studies/guys-and-stthomas-case-study-2 [Accessed 11 September 2017].

6 Guy's and St Thomas' Foundation Trust. About the Trust. (2015).

7 Guy's and St Thomas' Foundation Trust. Integrated quality and performance report December 2015, 2015.

8 Wade D. It's all about delivery - PRINCE2 Agile. Axelos, 2016.

9 Zhao P, Yoo I, Lavoie J, Lavoie B], Simoes E. Web-based medical appointment systems: a systematic review. J Med Internet Res 2017;19:e134.

10 McLean S, Gee M, Booth A et al. Targeting the use of reminders and notifications for uptake by populations (TURNUP): a systematic review and evidence synthesis. Southampton: NIHR Journals Library, 2014.

11 Muir S, Newell C, Griffiths ], et al. MotivATE: A pretreatment webbased program to improve attendance at UK outpatient services among adults with eating disorders. JMIR Res Protoc 2017;6:e146

12 Masoud T, Shah A, Joomun S, Shah A. Reducing DNA rates and increasing positive contacts in an outpatient chronic fatigue service. BMJ Qual Improv Rep 2017;6:u212876.w5262.

13 NHS England. NHS RightCare and the shift to value-based healthcare Introduction. 2017.

Address for correspondence: Mr Mark Bartlett, DrDoctor, ICNH Ltd, The Health Foundry, 1 Royal Street, London SE1 7LL, UK.

Email: Mark.bartlett@drdoctor.co.uk

\section{Assessing trainees in the workplace An e-learning module for secondary care doctors}

\author{
Three hours of CPD-approved interactive \\ learning covering: \\ $>$ feedback \\ > supervised learning events (SLES) \\ $>$ workplace-based assessments (WPBAs) \\ $>$ the role of the Annual Review of \\ Competence Progression (ARCP). \\ For more information please visit: \\ www.rcplondon.ac.uk/elearning
}

\section{Royal College of Physicians}

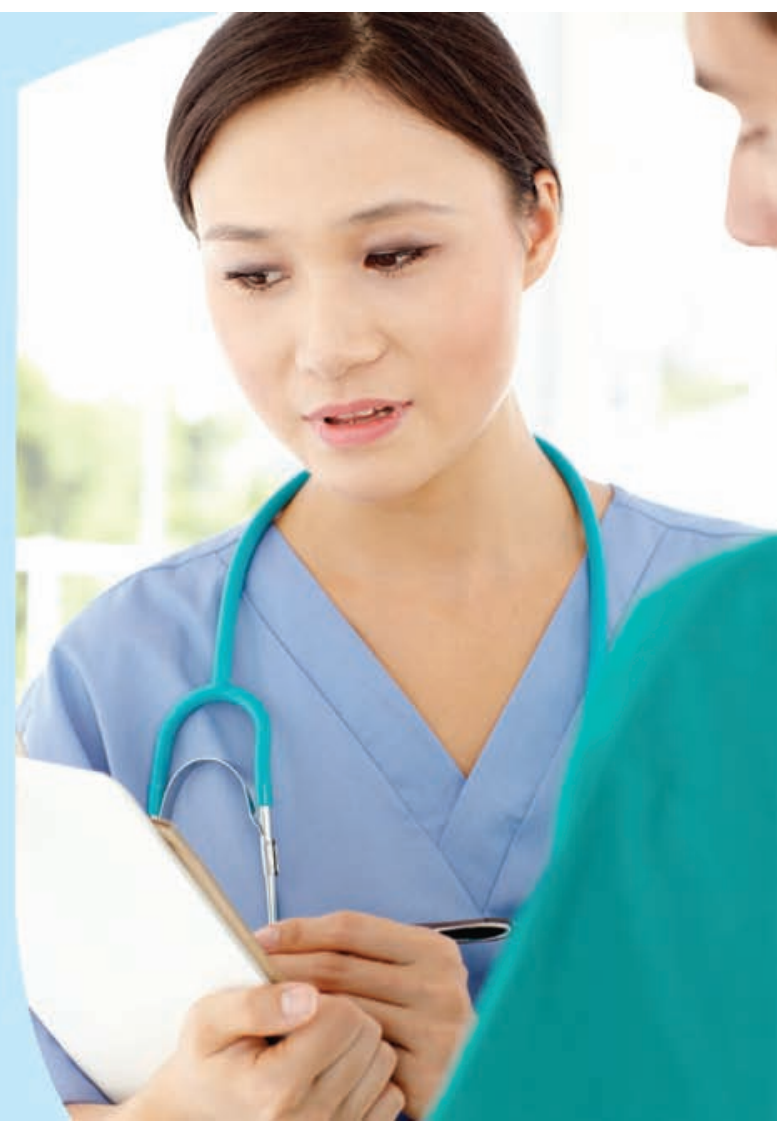

